\title{
ChemComm
}

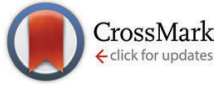

Cite this: Chem. Commun., 2016, 52,7114

Received 6th October 2015, Accepted 25th April 2016

DOI: $10.1039 / \mathrm{c} 5 \mathrm{cc} 08325 \mathrm{~g}$

www.rsc.org/chemcomm

\section{Structurally optimised BODIPY derivatives for imaging of mitochondrial dysfunction in cancer and heart cells $\dagger$}

\author{
Shubhanchi Nigam, ${ }^{\text {ab }}$ Benjamin P. Burke, ${ }^{\text {ab }}$ Laura H. Davies, ${ }^{c}{ }^{\text {Juozas Domarkas, }}{ }^{\text {bd }}$ \\ Jennifer F. Wallis, ${ }^{C}$ Paul G. Waddell,, Jennifer S. Waby, ${ }^{\mathrm{e}}$ David M. Benoit, ${ }^{\mathrm{C}}$ \\ Anne-Marie Seymour, ${ }^{\text {de }}$ Christopher Cawthorne, ${ }^{\text {be }}$ Lee J. Higham ${ }^{\star c}$ and \\ Stephen J. Archibald*ab
}

The structural features required for mitochondrial uptake of BODIPYbased optical imaging agents have been explored. The first derivatives of this class of dyes shown to have mitochondrial membrane potential-dependent uptake in both cancer and heart cells have been developed.

Mitochondrial membrane potential (MMP) plays a key role in cardiac failure and cancer. ${ }^{1}$ The electron transport chain contained within the impermeable inner mitochondrial membrane gives rise to a negative membrane potential (ca. $-150 \mathrm{mV}$ to $-170 \mathrm{mV}$ ) in healthy cells. ${ }^{2,3}$ In cancerous and ischaemic heart cells, mitochondrial dysfunction can substantially disrupt the $\mathrm{MMP}^{4,5}$ resulting in up to a tenfold increase in accumulation of MMP-dependent compounds. ${ }^{2,3}$ During apoptotic or necrotic cell death, complete membrane depolarisation occurs and no uptake is observed. ${ }^{6,7}$ Lipophilic cations carrying a positive charge can pass through lipid bilayers in the mitochondria and accumulate proportionally to the change in membrane potential gradient. $^{2}$ Optical agents have been developed for MMP-dependent imaging, with many derivatives based on rhodamine and tetramethylrosamine structures, with the highly effective Mitotracker ${ }^{\circledR}$ series now in common use. ${ }^{2,8}$

Boron-dipyrromethene (BODIPY) type structures are regularly used in optical biomedical imaging due to their tunable

\footnotetext{
${ }^{a}$ Department of Chemistry, University of Hull, Cottingham Road, Hull, HU6 7RX, UK. E-mail: S.J.Archibald@Hull.ac.uk

${ }^{b}$ Positron Emission Tomography Research Centre, University of Hull, Cottingham Road, Hull, HU6 $7 R X, U K$

${ }^{c}$ School of Chemistry, Newcastle University, Bedson Building, Newcastle upon Tyne, NE1 $7 R U, U K$

${ }^{d}$ Centre for Cardiovascular and Metabolic Research, University of Hull, Cottingham Road, Hull, HU6 $7 R X, U K$

${ }^{e}$ School of Biological, Biomedical and Environmental Sciences, University of Hull, Cottingham Road, HU6 $7 R X, U K$

$\dagger$ Electronic supplementary information (ESI) available: Synthetic details, fluorescence spectra, partition coefficient determination, confocal microscopy details, flow cytometry details and computational chemistry details. CCDC 1474862 . For ESI and crystallographic data in CIF or other electronic format see DOI: 10.1039/ c5cc08325g
}

wavelength emission, photostability, remarkable brightness and biological media compatibility. ${ }^{9-12}$ Recently, the use of BODIPYs has been further extended by the incorporation of the positron emitting radioisotope, fluorine-18, resulting in multimodal positron emission tomography (PET)/optical imaging agents. ${ }^{13-15}$ Formation of BODIPY based PET/optical multimodal imaging can be achieved via $\mathrm{B}-\mathrm{F}$ bond formation or modification of the structural backbone to incorporate the radiolabel.

To date, there have been no reports of BODIPY-based agents capable of imaging both cancer and heart cells in a MMPdependent manner. Phosphonium-based lipophilic cations have been receiving interest, most notably in the development of PET imaging agents. ${ }^{16-21}$ Recently, Yuan et al. described a BODIPY triphenylphosphonium-based lipophilic cation designed for multimodal imaging which did not show MMP-dependent mitochondrial uptake. $^{20}$ The development of MMP-dependent tracers would offer the capability for detection of mitochondrial dysfunction in cardiac disease and cancer together with the potential for monitoring of therapeutic responses.

Our approach in designing MMP dependent BODIPY derivatives is to form phosphonium cations directly on the lipophilic BODIPY backbone and study their chemical and biological characteristics. We have previously developed highly fluorescent, air-stable BODIPY-based phosphanes ${ }^{22}$ carrying either dicyclohexyl and diphenyl substituents. ${ }^{23}$ Methylation with methyl trifluoromethanesulfonate gave phosphonium derivatives $\mathbf{1}$ and 2 (see Fig. 1 and ESI $\dagger$ ).

Mitochondrial uptake studies of $\mathbf{1}$ and $\mathbf{2}$ were carried out using confocal microscopy in human breast cancer cells (MCF-7) and rat cardiomyocytes (H9c2). Mitotracker deep red (MDR) was used for dye co-localisation studies as its emission wavelength $(665 \mathrm{~nm})$ is sufficiently different from that of $\mathbf{1}$ and $2(532 \mathrm{~nm})$ to allow independent detection. Confocal microscopy confirmed mitochondrial localisation of both 1 and 2 (see Fig. 2 and ESI $\dagger$ for further details). Overlap coefficients identified that both tracers showed similarly high mitochondrial localisation in H9c2 cells, however the overlap coefficient was $36 \%$ higher in MCF-7 cells for 1, indicating that it has significantly greater potential as a specific imaging agent. 


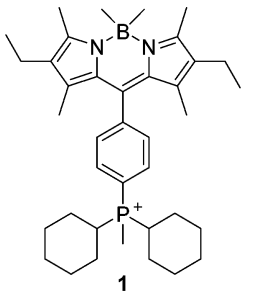

-OTf

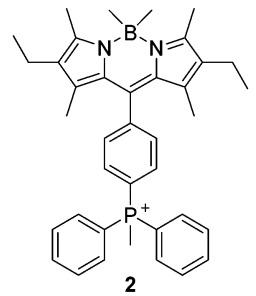

OTf

Fig. 1 Chemical structures of phosphonium BODIPY derivatives 1 and 2 .
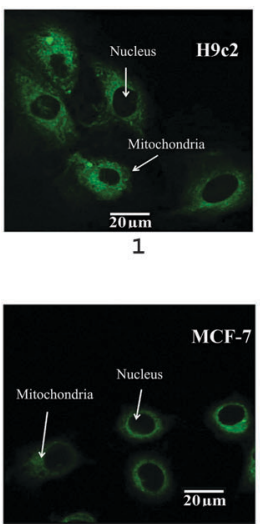

1

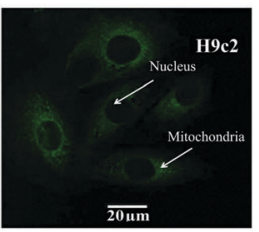

2

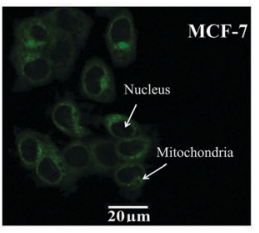

2

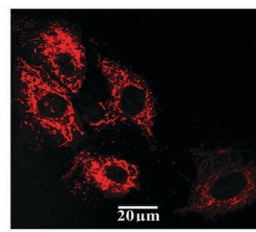

MDR

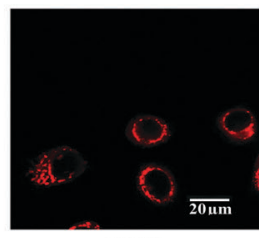

MDR

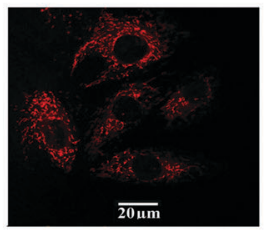

MDR

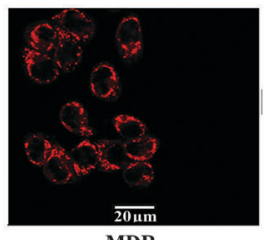

MDR

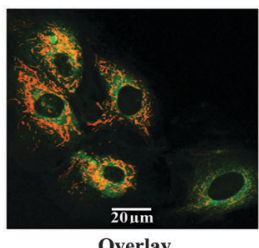

Overlay
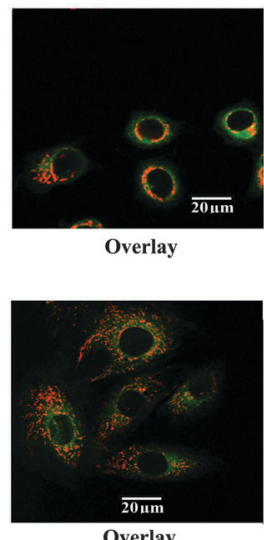

Overlay

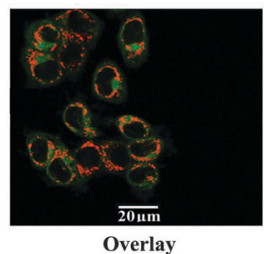

Fig. 2 Confocal microscopy of 1 and 2 (left) in MCF-7 and H9c2 cell lines with mitotracker deep red (MDR, centre), overlaid mitochondrial co-localisation (right).

Imaging agents with MMP-dependent uptake offer an ideal tool to probe mitochondrial function. To establish the mitochondrial membrane potential specific uptake of $\mathbf{1}$ and 2, a flow cytometry study was carried out in the presence of carbonyl cyanide $m$-chlorophenylhydrazone (CCCP), a protonophore which eliminates the mitochondrial membrane potential (see Fig. 3 and ESI $\dagger$ for further details). ${ }^{24,25}$ Both tracers showed a decrease in mean fluorescent intensity (MFI) upon CCCP induced MMP depolarisation in both cell lines. 1 showed a greater than $70 \%$ decrease in MCF-7 and H9c2 cell lines, whereas 2 showed a smaller $38 \%$ and $58 \%$ decrease in MCF-7 and $\mathrm{H} 9 \mathrm{c} 2$ cells respectively.

The vast majority of lipophilic cations used in molecular imaging are based on triphenylphosphonium ions. ${ }^{3,16,18,19}$ In this study,
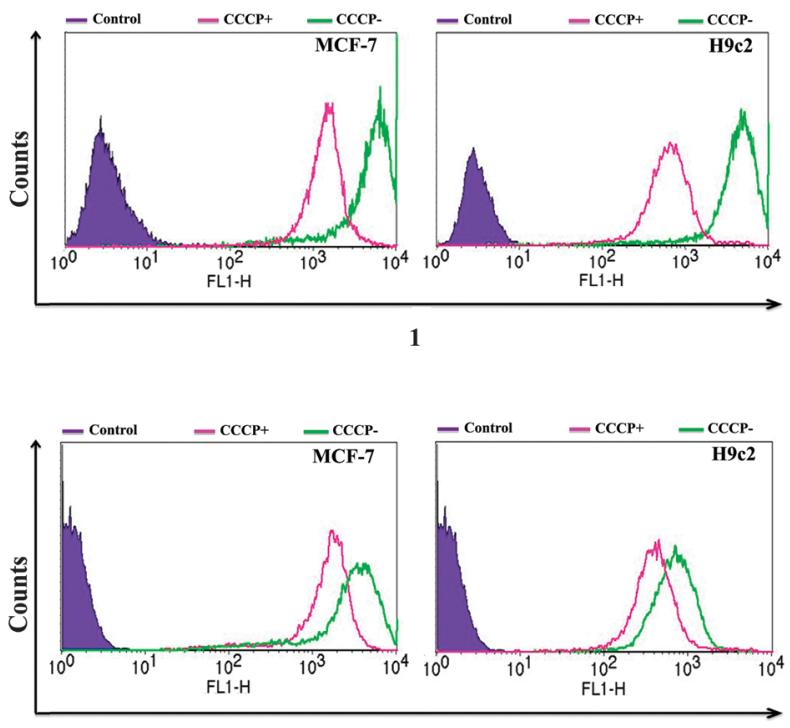

2

Fig. 3 Flow cytometry studies of $\mathbf{1}$ and $\mathbf{2}$ in MCF-7 (left) and H9c2 (right) cells without (green) and with (pink) the presence of mitochondrial membrane depolarising agent (CCCP) to assess mitochondrial membrane potential uptake.

we have designed and synthesised a triphenylphosphonium BODIPY structure (2) along with a cyclohexyl derivative (1) to explore the structural features necessary for MMP-dependent uptake. Both the mitochondrial localisation determined by confocal microscopy and the flow cytometry MMP-dependent uptake studies demonstrate that the replacement of two phenyl groups for two cyclohexyl groups cause an increase in MMP dependent, mitochondrial specific uptake. DFT calculations were carried out on both structures to determine the degree of delocalisation of electron density. Our calculations show (see Fig. 4 and ESI $\dagger$ for further details) that the charge is localised on the phosphorus atom for the cyclohexyl derivative (1) while it is mainly delocalised on the aromatic ligands for the triphenylphosphonium structure (2). The effect of the degree of lipophilicity on the uptake of lipophilic cations has been partially explored in previous studies, generally when describing selectivity between cancer and cardiac uptake. ${ }^{16}$ The lipophilicity $(\log P)$ values of 1 and 2 were determined to be 3.19 and 2.80 respectively, revealing a significant increase in lipophilicity for the cyclohexyl derivative.

To validate the strategy and open opportunities for future PET/optical imaging agents, we have replaced methyl trifluoromethanesulfonate in the final step methylation reaction of the dicyclohexyl derivative with 1-bromo-4-fluorobutane. The structure of the formed product, 3 , was confirmed by NMR and $\mathrm{X}$-ray crystallography (see ESI $\dagger$ ) and is the cold standard of a potential future radiolabelled product. The fluorine-18 analogue can be prepared via conventional nucleophilic substitution in a one step procedure or directly from 1 in a 2-step radiolabelling protocol. $^{26,27}$

Flow cytometry studies on 3 , carried out using an identical protocol to that used to analyse $\mathbf{1}$ and 2, demonstrated that this fluoride substituted derivative behaves in an analogous fashion. 

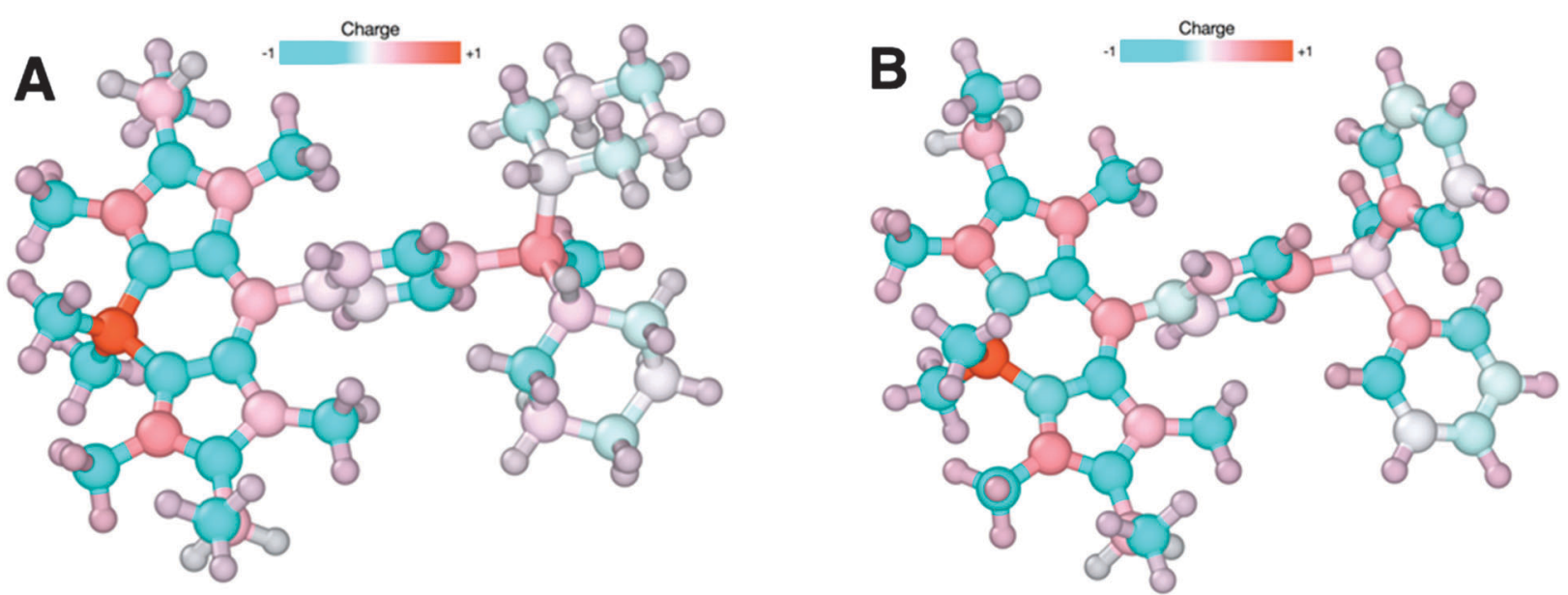

Fig. 4 Charge distribution for structure (1) (A, left) and structure (2) (B, right) computed at the TPSS-D3/def2-TZVPP level of theory using their fully optimised geometries. The atomic charges are determined using the CHELPG method and represented using a colour scheme where red indicates a $+1 e$ nominal charge and blue a -1e nominal charge. Note the concentration of charge around phosphorus in (A) which is not seen in (B).

Replacement of the methyl group in $\mathbf{1}$ with a fluorobutyl leads to an increase in MMP dependent uptake, with CCCP causing decreases in uptake of $84 \%$ and $83 \%$ in MCF-7 and H9c2 cells respectively (see Fig. 5), thus validating this approach for the design of multimodal optical/PET imaging agents.

In conclusion, we have developed novel BODIPY based agents for mitochondrial imaging. These agents, for the first time, have been shown to be taken up in mitochondria in a mitochondrial membrane potential-dependent manner in both cancer and heart cells. The new structural class, with the phosphonium grafted directly on the BODIPY backbone, offers the opportunity to reduce non-specific binding by optimising the lipophilicity of a dicyclohexyl derviative with shielded localised charge. This work validates the structural features required to develop MMP-dependent PET/ optical multimodal imaging via fluorine-18 radiolabelling. Studies are currently underway to develop these agents for in vivo use.
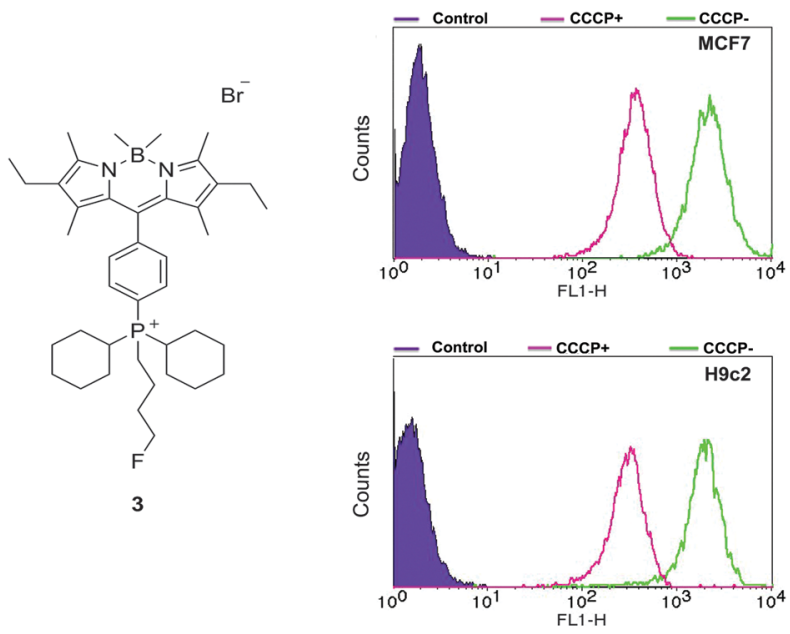

Fig. 5 Chemical structure of fluorine substituted dicyclohexyl BODIPY derivative 3 and flow cytometry in MCF-7 (top) and H9c2 (bottom) cells without (green) and with (pink) the presence of mitochondrial membrane depolarising agent (CCCP) to assess mitochondrial membrane potential uptake.
We gratefully acknowledge the Daisy Appeal Charity for funding (Grant: DAhul0211), the University of Hull for a fee reduction scholarship for SN, Newcastle University for a studentship LHD, EPSRC and High Force Research Ltd for financial support of JFW and EPSRC for a LJH Fellowship (EP/G005206/1). We thank Dr Assem Allam for his generous donation and ongoing support to the PET Research Centre at the University of Hull. The authors wish to thank the EPSRC National Mass Spectrometry Service at Swansea.

\section{Notes and references}

1 S. Fulda, L. Galluzzi and G. Kroemer, Nat. Rev. Drug Discovery, 2010, 9, 447-464.

2 M. P. Murphy, Biochim. Biophys. Acta, 2008, 1777, 1028-1031.

3 J. S. Modica-Napolitano and J. R. Aprille, Adv. Drug Delivery Rev., 2001, 49, 63-70.

4 J. S. Modica-Napolitano and J. R. Aprille, Cancer Res., 1987, 47, 4361-4365.

5 B. Kadenbach, R. Ramzan, R. Moosdorf and S. Vogt, Mitochondrion, 2011, 11, 700-706.

6 H. W. Strauss and H. Schoeder, J. Am. Coll. Cardiol. Img., 2012, 5, 293-296.

7 V. Ganitkevich, S. Reil, B. Schwethelm, T. Schroeter and K. Benndorf, Circ. Res., 2006, 99, 165-171.

8 M. Poot, Y. Z. Zhang, J. A. Kramer, K. S. Wells, L. Jones, D. K. Hanzel, A. G. Lugade, V. L. Singer and R. P. Haughland, J. Histochem. Cytochem., 1996, 44, 1363-1372.

9 G. Ulrich, R. Ziessel and A. Harriman, Angew. Chem., Int. Ed., 2008, 47, 1184-1201.

10 N. Boens, V. Leen and W. Dehaen, Chem. Soc. Rev., 2012, 41, 1130-1172.

11 J. Karolin, L. B. A. Johansson, L. Strandberg and T. Ny, J. Am. Chem. Soc., 1994, 116, 7801-7806.

12 L. H. Davies, B. B. Kasten, P. D. Benny, R. L. Arrowsmith, H. Ge, S. I. Pascu, S. W. Botchway, W. Clegg, R. W. Harrington and L. J. Higham, Chem. Commun., 2014, 50, 15503-15505.

13 Z. Li, T. P. Lin, S. Liu, C. W. Huang, T. W. Hudnall, F. P. Gabbai and P. S. Conti, Chem. Commun., 2011, 47, 9324-9326.

14 J. A. Hendricks, E. J. Keliher, D. Wan, S. A. Hilderbrand, R. Weissleder and R. Mazitschek, Angew. Chem., Int. Ed., 2012, 51, 4603-4606.

15 B. P. Burke, G. S. Clemente and S. J. Archibald, Contrast Media Mol. Imaging, 2015, 10, 96-110.

16 Y. Zhou and S. Liu, Bioconjugate Chem., 2011, 22, 1459-1472.

17 X. Yan, Y. Zhou and S. Liu, Theranostics, 2012, 2, 988-998.

18 D.-Y. Kim, H.-S. Kim, L. Uyenchi Nguyen, S. N. Jiang, H.-J. Kim, K.-C. Lee, S.-K. Woo, J. Chung, H.-S. Kim, H.-S. Bom, K.-H. Yu and J.-J. Min, J. Nucl. Med., 2012, 53, 1779-1785. 
19 Z. Zhao, Q. Yu, T. Mou, C. Liu, W. Yang, W. Fang, C. Peng, J. Lu, Y. Liu and X. Zhang, Mol. Pharmaceutics, 2014, 11, 3823-3831.

20 H. Yuan, H. Cho, H. H. Chen, M. Panagia, D. E. Sosnovik and L. Josephson, Chem. Commun., 2013, 49, 10361-10363.

21 B. P. Burke, P. Greenman, A. M. Smith and S. J. Archibald, Acta Crystallogr., Sect. E: Struct. Rep. Online, 2012, 68, 03202.

22 L. H. Davies, B. Stewart, R. W. Harrington, W. Clegg and L. J. Higham, Angew. Chem., Int. Ed., 2012, 51, 4921-4924.
23 L. H. Davies, R. W. Harrington, W. Clegg and L. J. Higham, Dalton Trans., 2014, 43, 13485-13499.

24 H. Steen, J. G. Maring and D. K. F. Meijer, Biochem. Pharmacol., 1993, 45, 809-818.

25 M. L. R. Lim, T. Minamikawa and P. Nagley, FEBS Lett., 2001, 503, 69-74. 26 P. W. Miller, N. J. Long, R. Vilar and A. D. Gee, Angew. Chem., Int. Ed., 2008, 47, 8998-9033.

27 F. Buckingham and V. Gouverneur, Chem. Sci., 2016, 7, 1645-1652. 